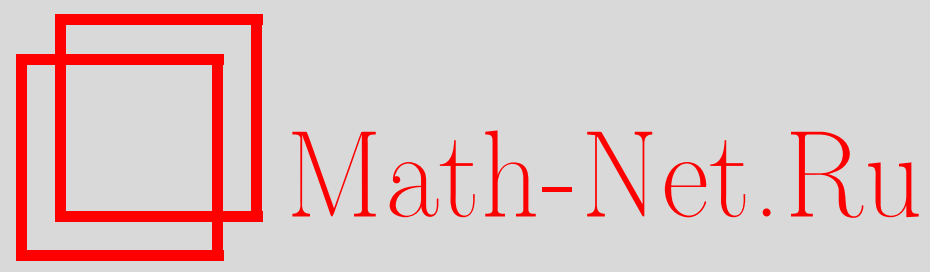

П. В. Довбуш, Критерий полиномиальности для целых функций многих комплексных переменных, Матем. заметки, 1999, том 66, выпуск 4, 500-502

DOI: https://doi.org/10.4213/mzm3986

Использование Общероссийского математического портала Math-Net.Ru подразумевает, что вы прочитали и согласны с пользовательским соглашением http://www . mathnet.ru/rus/agreement

Параметры загрузки:

IP: 18.208 .226 .222

26 апреля 2023 г., 15:16:22

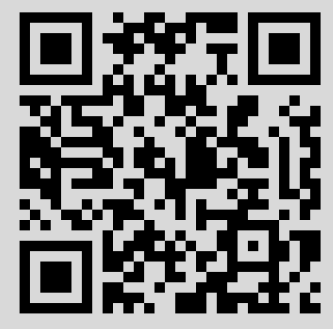




\section{КРИТЕРИЙ ПОЛИНОМИАЛЬНОСТИ ДЛЯ ЦЕЛЫХ ФУНКЦИЙ МНОГИХ КОМПЛЕКСНЫХ ПЕРЕМЕННЫХ}

\section{П. В. Довбуш}

Доказан "радиальный” критерий полиномиальности для целых функций многих комплексных переменных.

Библиография: 3 названия.

$\Phi$ ункция $f: \mathbb{C}^{n} \rightarrow \mathbb{C}, n \geqslant 1$, голоморфная во всем $n$-мерном комплексном пространстве $\mathbb{C}^{n}$, называется иелой функиией.

Если целая функция $f: \mathbb{C}^{n} \rightarrow \mathbb{C}, n \geqslant 1$, имеет однородное разложение

$$
f(z)=\sum_{k=0}^{\infty} P_{k}(z)
$$

в котором $P_{k}$ есть однородные многочлены в $\mathbb{C}^{n}$ степени $k$, то радиальная производная $\mathscr{R} f$ определяется по формуле

$$
\mathscr{R} f(z)=\sum_{k=1}^{\infty} k P_{k}(z) .
$$

В дальнейшем через $l_{\xi}$ будем обозначать комплексную прямую в $\mathbb{C}^{n}$, проходящую через нуль и фиксированную точку $\xi \in S$, где $S$ - единичная сфера в $\mathbb{C}^{n}$ с центром в нуле.

Радиальная производная $\mathscr{R} f$ связана с производной сужения функции $f$ на комплексную прямую $l_{\xi}(\lambda)=\{z=\lambda \xi, \lambda \in \mathbb{C}, \xi \in S\}$ следующим соотношением:

$$
\mathscr{R} f\left(l_{\xi}(\lambda)\right)=\lambda f^{\prime}\left(l_{\xi}(\lambda)\right), \quad \lambda \in \mathbb{C}, \quad \xi \in S,
$$

где штрих означает производную по $\lambda$.

Имеет место

Теорема. Целая функиия $f: \mathbb{C}^{n} \rightarrow \mathbb{C}, n \geqslant 1$, является многочленом тогда и только тогда, когда для любой комплексной прямой $l \subseteq \mathbb{C}^{n}$, проходящей через начало координат,

$$
\varlimsup_{|\lambda| \rightarrow \infty} \frac{|\mathscr{R} f(l(\lambda))|}{1+|f(l(\lambda))|^{2}}<\infty .
$$


ДокАЗАТЕЛЬСТво. Необходимость условия (2) проверяется непосредственно, поэтому покажем его достаточность.

Сначала рассмотрим случай $n=1$.

Если целая функция $f$ не является тождественной постоянной, то возможны два случая: либо у функции $f$ в бесконечности имеется полюс, либо - существенная особенность.

Предположим, что имеет место второй случай. Тогда у функции $g(z)=f(1 / z)$ в нуле будет существенная особенность. Из условий (1) и (2) следует, что в проколотом единичном круге $U_{*}=\{z \in \mathbb{C}, 0<|z|<1\}$ вьполняется неравенство

$$
\frac{|z| \cdot\left|g^{\prime}(z)\right|}{1+|g(z)|^{2}}<O(1)
$$

Введем в рассмотрение семейство функций $\mathscr{F}=\left\{g_{k}(z)\right\}$, где функции $g_{k}(z)=g\left(z / 2^{k}\right)$, $k=1,2, \ldots$, определены и голоморфны в $U_{*}$.

Непосредственно проверяется, что

$$
\frac{\left|g_{k}^{\prime}(z)\right|}{1+\left|g_{k}(z)\right|^{2}}=\frac{1}{|z|} \frac{\left(|z| / 2^{k}\right)\left|g^{\prime}\left(z / 2^{k}\right)\right|}{1+\left|g\left(z / 2^{k}\right)\right|^{2}}
$$

для всех $z \in U_{*}$.

Из этого равенства, неравенства (3) и критерия Марти (см., например, [1, с. 238]) заключаем, что $\mathscr{F}$ - нормальное в $U_{*}$ семейство. Получили противоречие с теоремой Монтеля (см. [2, с. 71]), согласно которой семейство $\mathscr{F}$ нормальньм в $U_{*}$ быть не может, так как функция $g$ в нуле имеет существенную особенность. Следовательно, целая функция $f$ в бесконечности имеет полюс и, значит, является многочленом.

Рассмотрим теперь случай $n>1$.

Из условия (2) и сказанного вьше следует, что сужение $f$ на любую комплексную прямую $l_{\xi}$ является многочленом. Если $f(0)=0$, то найдется комплексная прямая $l_{\zeta}$ такая, что многочлен $f\left(l_{\zeta}(\lambda)\right)$ тождественно равен 0 , и тогда его степень не определена. Чтобы этого не произошло в случае, когда $f(0)=0$, вместо функции $f$ рассмотрим функцию $f-1$. Функция $f-1$ также удовлетворяет условию (2), это следует из определения радиальной производной и элементарных неравенств

$$
\frac{1}{3}<\frac{1+|f-1|^{2}}{1+|f|^{2}}<3
$$

(Правое неравенство следует из того, что $1+|f-1|^{2} \leqslant 1+|f|^{2}+2|f|+1<3\left(1+|f|^{2}\right.$ ), а левое получается из правого заменой $f$ на $1-f$.) Поэтому с самого начала можем считать, что $f(0) \neq 0$.

Положим $E_{m}=\left\{\xi \in S: f\left(l_{\xi}(\lambda)\right)\right.$ - многочлен степени не выше $\left.m\right\}, m=1,2, \ldots$.

Покажем, что множества $E_{m}$ замкнуты в $S$. Пусть $\left\{\xi_{k}\right\}$ - последовательность точек из $E_{m}$, сходящаяся к точке $\xi \in S$. Многочлены $f\left(l_{\xi_{k}}(\lambda)\right)$ имеют степени не выше $m$ и равномерно на компактных подмножествах $\mathbb{C}$ сходятся к функции $f\left(l_{\xi}(\lambda)\right)$. Отсюда и из теоремы Вейерштрасса (см., например, $[3$, c. 34$])$ следует, что $f\left(l_{\xi}(\lambda)\right)$ является 
многочленом степени не вьше $m$; следовательно, по определению множества $E_{m}$ точка $\xi \in E_{m}$ и, значит, множество $E_{m}$ замкнуто в $S$.

Очевидно, что

$$
S=\bigcup_{m=1}^{\infty} E_{m}
$$

Но по теореме Бэра $S$ нельзя представить в виде счетного объединения нигде не плотных множеств, поэтому для некоторого $N$ множество $E_{N}$ содержит непустое открытое множество $S_{1} \subset S$. Поэтому найдется некоторьй круговой конус $K$, на котором $f$ есть многочлен степени не вьше $N$. Отсюда и из теоремы единственности в $\mathbb{C}^{n}$ заключаем, что $f$ является многочленом в $\mathbb{C}^{n}$.

\section{СПИСОК ЦИТИРОВАННОЙ ЛИТЕРАТУРЫ}

[1] Хейман У. Мероморфные функции. М.: Мир, 1966.

[2] Голузин Г. М. Геометрическая теория функций комплексного переменного. М.: Наука, 1966.

[3] Шабат Б. В. Введение в комплексный анализ. Т. 2. М.: Наука, 1976.

Институт математики АН Молдовы, г. Кишинев

Поступило

E-mail: 15dovb@matem.moldova.su 